\title{
Measuring charge fluctuations in high-energy nuclear collisions
}

\author{
Stanisław Mrówczyński* \\ Sottan Institute for Nuclear Studies, \\ ul. Hoża 69, PL - 00-681 Warsaw, Poland \\ and Institute of Physics, Świętokrzyska Academy, \\ ul. Świętokrzyska 15, PL - 25-406 Kielce, Poland
}

(1-st July 2002)

\begin{abstract}
Various measures of charge fluctuations in heavy-ion collisions are discussed. Advantages of the $\Phi-$ measure are demonstrated and its relation to other fluctuation measures is established. To get the relation, $\Phi$ is expressed through the moments of multiplicity distribution. We study how the measures act in the case of a 'background' model which represents the classical hadron gas in equilibrium. The model assumes statistical particle production constrained by charge conservation. It also takes into account both the effect of incomplete experimental apparatus acceptance and that of tracking inefficiency. The model is shown to approximately agree with the PHENIX and preliminary STAR data on the electric charge fluctuations. Finally, 'background-free' measures are discussed.
\end{abstract}

PACS: 25.75.-q, 24.60.Ky, 24.60.-k

Keywords: Relativistic heavy-ion collisions; Statistical model; Fluctuations

\section{INTRODUCTION}

Fluctuations of strange, baryonic and electric charges studied on event-by-event basis have been repeatedly argued to provide dynamical information on high-energy heavy-ion collisions. Jeon and Koch [1] have suggested to study the fluctuations of the ratio of positive to negative pions in order to measure the number of $\rho$ and $\omega$ resonances after hadronization. Gavin and Pruneau [2] have found that the baryon number fluctuations are very sensitive to the degree of chemical equilibration of the systems produced in heavy-ion collisions at RHIC and LHC. Gavin with collaborators 3. 1. have also suggested that the extraordinary baryon fluctuations can serve as a signal of the QCD tricritical point [5]. Jeon and Koch [9] and Asakwa, Heinz and Müller [10] have observed that the fluctuations of baryonic and electric charge are significantly smaller in the equilibrium quark-gluon plasma than in the hadron gas. Assuming that the fluctuations created in the quark phase survive the hadronization, the charge fluctuations normalized to the entropy, which is also assumed to be conserved, can be exploited as an indicator of the quark-gluon plasma formation in nuclear collisions [9,10]. The idea has been further discussed in [11 18].

The NA49 measurement [19,20] of the $K / \pi$ ratio at the SPS collision energy is somewhat discouraging. It suggests that the fluctuations in the central collisions are mostly of trivial statistical character. The conclusion has been theoretically analyzed in [1,21,22]. The PHENIX [23] and preliminary STAR [24 results on charge fluctuations show that statistical noise also dominates at RHIC energies. Therefore, one faces a problem how to extract a small contribution of 'dynamical' fluctuations of interest from the statistical background which, unfortunately, strongly depends on the collision centrality. Among other methods, the problem can be solved by means of the so-called $\Phi-$ measure [25] which has been successfully applied to the $p_{T}$-fluctuations [26]. $\Phi$ equals zero when inter-particle correlations are absent. It also eliminates 'geometrical' fluctuations due to the impact parameter variation. Therefore, $\Phi$ is 'deaf' to the statistical noise and 'blind' to the collision centrality.

Applicability of $\Phi$ to the fluctuations of chemical composition of the hadronic system produced in nuclear collisions has been already discussed in 27,28]. In this paper we advocate usefulness of $\Phi$ in studies of charge fluctuations which are obviously related to the chemical fluctuations. Advantages of $\Phi$ in such studies have been already demonstrated in a very recent paper [29]. Here, we also discuss another measure denoted as $\Gamma$, which is closely related to $\Phi$. However, $\Gamma$ is sensitive not only to the dynamical fluctuations, as is $\Phi$, but to the statistical fluctuations as well. We express

*Electronic address: mrow@fuw.edu.pl 
$\Phi$ and $\Gamma$ through the moments of multiplicity distributions and then we compare them to the fluctuation measures suggested by other authors [1,9, 10,21,24. We also compute the measures for a 'background' model where the particle production is mostly statistical but constrained by charge conservation. The model represents the classical hadron gas in equilibrium, where hadron resonances are neglected. After taking into account a finite detector acceptance, which strongly reduces the effect of charge conservation, the model's predictions are compared to the PHENIX [23] and preliminary STAR [24] data on the electric fluctuations [24]. At the end, we discuss the measures where the background fluctuations, i.e., those given by the 'background' model, are eliminated. The measures are free of trivial effects caused by the charge conservation and finite detector acceptance.

\section{II. $\Phi-$ AND $\Gamma-$ MEASURE}

Let us introduce the measure $\Phi$ which describes the correlations (or fluctuations) of a single particle variable $x$. Here, $x$ is identified with the particle electric, baryonic or any other charge $q$. One defines a single-particle variable

$z \stackrel{\text { def }}{=} x-\bar{x}$ with the overbar denoting averaging over a single particle inclusive distribution. One easily observes that $\bar{z}=0$. Further, we introduce the event variable $Z$, which is a multiparticle analog of $z$, defined as $Z \stackrel{\text { def }}{=} \sum_{i=1}^{N}\left(x_{i}-\bar{x}\right)$, where the summation runs over particles in a given event. By construction, $\langle Z\rangle=0$, where $\langle\ldots\rangle$ represents averaging over events. Finally, the $\Phi$-measure is defined in the following way:

$$
\Phi \stackrel{\text { def }}{=} \sqrt{\frac{\left\langle Z^{2}\right\rangle}{\langle N\rangle}}-\sqrt{\overline{\overline{z^{2}}}} .
$$

It is evident that $\Phi=0$, when no inter-particle correlations are present. The measure also possesses a less trivial property. Namely, $\Phi$ is independent of the distribution of the number of particle sources if the sources are identical and independent from each other [25,30]. Thus, the $\Phi$-measure is 'blind' to the impact parameter variation as long as the 'physics' does not change with the collision centrality. In particular, the $\Phi$ is independent of the impact parameter if the nucleus-nucleus collision is a simple superposition of nucleon-nucleon interactions.

As in the case of chemical fluctuations [28], we are going to express $\Phi$ through the moments of multiplicity distribution. Then, $\Phi$ can be compared to other fluctuation measures which are usually defined in this way. We first consider a system of particles with two different values of charge. In principle, the system might be multi-component but only two charged components are taken into account. Then, $x$ equals either $q_{1}$ or $q_{2}$. The inclusive averages of $x$ and $x^{2}$ read

$$
\bar{x}=q_{1} P_{1}+q_{2} P_{2}, \quad \overline{x^{2}}=q_{1}^{2} P_{1}+q_{2}^{2} P_{2},
$$

where the probabilities to find a particle with $q_{1}$ and $q_{2}$, respectively, are

$$
P_{i}=\frac{\left\langle N_{i}\right\rangle}{\langle N\rangle}, \quad i=1,2
$$

with $N_{i}$ being the number of particles with charge $q_{i}$ and $N \equiv N_{1}+N_{2}$. One easily finds that

$$
\overline{z^{2}}=\left(q_{1}-q_{2}\right)^{2} \frac{\left\langle N_{1}\right\rangle\left\langle N_{2}\right\rangle}{\langle N\rangle^{2}} .
$$

Using the relation

$$
Z=Q-\frac{\langle Q\rangle}{\langle N\rangle} N
$$

where $Q=q_{1} N_{1}+q_{2} N_{2}$ is the system charge, we get $\langle Z\rangle=0$ and

$$
\frac{\left\langle Z^{2}\right\rangle}{\langle N\rangle}=\frac{\left(q_{1}-q_{2}\right)^{2}}{\langle N\rangle^{3}}\left[\left\langle N_{1}\right\rangle^{2}\left\langle N_{2}^{2}\right\rangle+\left\langle N_{1}^{2}\right\rangle\left\langle N_{2}\right\rangle^{2}-2\left\langle N_{1}\right\rangle\left\langle N_{2}\right\rangle\left\langle N_{1} N_{2}\right\rangle\right],
$$

which can be rewritten as 


$$
\begin{aligned}
\frac{\left\langle Z^{2}\right\rangle}{\langle N\rangle}=\left(q_{1}-q_{2}\right)^{2} \frac{\left\langle N_{1}\right\rangle^{2}\left\langle N_{2}\right\rangle^{2}}{\langle N\rangle^{3}}\left[\frac{\left\langle N_{1}^{2}\right\rangle-\left\langle N_{1}\right\rangle^{2}}{\left\langle N_{1}\right\rangle^{2}}\right. & +\frac{\left\langle N_{2}^{2}\right\rangle-\left\langle N_{2}\right\rangle^{2}}{\left\langle N_{2}\right\rangle^{2}} \\
& \left.-2 \frac{\left\langle N_{1} N_{2}\right\rangle-\left\langle N_{1}\right\rangle\left\langle N_{2}\right\rangle}{\left\langle N_{1}\right\rangle\left\langle N_{2}\right\rangle}\right] .
\end{aligned}
$$

The fluctuation measure $\Phi$ is completely determined by Eqs. (2, 3). If the particle distributions are poissonian and independent from each other i.e.

$$
\begin{aligned}
\left\langle N_{i}^{2}\right\rangle-\left\langle N_{i}\right\rangle^{2} & =\left\langle N_{i}\right\rangle, \quad i=1,2 \\
\left\langle N_{1} N_{2}\right\rangle & =\left\langle N_{1}\right\rangle\left\langle N_{2}\right\rangle
\end{aligned}
$$

one notices that

$$
\frac{\left\langle Z^{2}\right\rangle}{\langle N\rangle}=\left(q_{1}-q_{2}\right)^{2} \frac{\left\langle N_{1}\right\rangle\left\langle N_{2}\right\rangle}{\langle N\rangle^{2}}
$$

and $\Phi=0$. In general, $\Phi$ vanishes when particles are independent form each other and the event's charge per particle is independent of the event's multiplicity.

If one studies a system with particles carrying more than two different values of a given charge, Eqs. (2,3) have to be generalized. We first consider the generalization to the three-component system, such as that of positive $(q=1)$, negative $(q=-1)$ and neutral $(q=0)$ hadrons. Although the neutral particles do not contribute to the system's charge, they do contribute to the fluctuations measured by $\Phi$. After rather lengthy calculations one finds

$$
\overline{z^{2}}=\left(q_{1}-q_{2}\right)^{2} \frac{\left\langle N_{1}\right\rangle\left\langle N_{2}\right\rangle}{\langle N\rangle^{2}}+\left(q_{1}-q_{3}\right)^{2} \frac{\left\langle N_{1}\right\rangle\left\langle N_{3}\right\rangle}{\langle N\rangle^{2}}+\left(q_{2}-q_{3}\right)^{2} \frac{\left\langle N_{2}\right\rangle\left\langle N_{3}\right\rangle}{\langle N\rangle^{2}}
$$

and

$$
\frac{\left\langle Z^{2}\right\rangle}{\langle N\rangle}=\frac{\left(q_{1}-q_{2}\right)^{2}}{\langle N\rangle^{3}} A_{12}+\frac{\left(q_{1}-q_{3}\right)^{2}}{\langle N\rangle^{3}} A_{13}+\frac{\left(q_{2}-q_{3}\right)^{2}}{\langle N\rangle^{3}} A_{23},
$$

where $N \equiv N_{1}+N_{2}+N_{3}$ and

$$
\begin{aligned}
A_{12} & \equiv\left\langle N_{1}^{2}\right\rangle\left(\left\langle N_{2}\right\rangle^{2}+\left\langle N_{2}\right\rangle\left\langle N_{3}\right\rangle\right)+\left\langle N_{2}^{2}\right\rangle\left(\left\langle N_{1}\right\rangle^{2}+\left\langle N_{1}\right\rangle\left\langle N_{3}\right\rangle\right)-\left\langle N_{1}\right\rangle\left\langle N_{2}\right\rangle\left\langle N_{3}^{2}\right\rangle \\
& \left.-\left\langle N_{1} N_{2}\right\rangle\left(2\left\langle N_{1}\right\rangle\left\langle N_{2}\right\rangle+\left\langle N_{2}\right\rangle\left\langle N_{3}\right\rangle+\left\langle N_{1}\right\rangle\left\langle N_{3}\right\rangle+\left\langle N_{3}\right\rangle^{2}\right\rangle\right) \\
& +\left\langle N_{2} N_{3}\right\rangle\left(\left\langle N_{1}\right\rangle^{2}-\left\langle N_{1}\right\rangle\left\langle N_{2}\right\rangle+\left\langle N_{1}\right\rangle\left\langle N_{3}\right\rangle\right)+\left\langle N_{1} N_{3}\right\rangle\left(\left\langle N_{2}\right\rangle^{2}-\left\langle N_{1}\right\rangle\left\langle N_{2}\right\rangle+\left\langle N_{2}\right\rangle\left\langle N_{3}\right\rangle\right) .
\end{aligned}
$$

$A_{13}$ can be found from $A_{12}$ by swapping indices $2 \leftrightarrow 3$ and $A_{23}$ coincides with $A_{13}$ when $1 \leftrightarrow 2$. One easily shows that for the poissonian distribution (5), $\left\langle Z^{2}\right\rangle /\langle N\rangle=\overline{z^{2}}$ and $\Phi=0$.

The formulas (6.76) can be further generalized to a system of four or higher number of particle species, such as the quark-gluon plasma. While the modifications of Eqs. (6.7) are obvious, Eq. (8) should be understood in such a way that $N_{3}$ represents all particles other than those carrying charges $q_{1}$ or $q_{2}$.

The $\Phi$-measure has been designed to look for 'dynamical' fluctuations. As seen, it vanishes when the fluctuations are of simple statistical origin. However, for the theoretical suggestion [9,10] the fluctuation magnitude is the main issue. It has been observed by the authors of $[9.10]$ that the statistical fluctuations generated in the quark-gluon phase are significantly smaller than those in the hadron gas. Therefore, if the quark-gluon plasma fluctuations are frozen due to the fast longitudinal expansion of the system the fluctuations observed at the hadron phase are significantly smaller than the statistical fluctuations characteristic for the hadron gas. Then, instead of the $\Phi$-measure defined by Eq. (1), one cane use $\left\langle Z^{2}\right\rangle /\langle N\rangle$, which is, as is $\Phi$, insensitive to the distribution of the independent particle sources. Therefore, we define a measure

$$
\Gamma \stackrel{\text { def }}{=} \frac{1}{\langle N\rangle}\left\langle\left(Q-\frac{\langle Q\rangle}{\langle N\rangle} N\right)^{2}\right\rangle=\frac{\left\langle Z^{2}\right\rangle}{\langle N\rangle},
$$

which was introduced in the very first paper on $\Phi$ [25]. As discussed below, $\Gamma$, which measures both the dynamical and statistical fluctuations, can be very useful in the experimental data analysis. 


\section{OTHER MEASURES}

In this section we compare $\Phi$ and $\Gamma$ to other measures of charge fluctuations. We limit the comparison to the case of two-component system. The charge fluctuations can be studied by means of the ratio of the multiplicities of particles of different charges, $R=N_{1} / N_{2}$ [1] 9]. One finds [1]21] that to the second order in the fluctuations of numbers of particles

$$
\left\langle R^{2}\right\rangle-\langle R\rangle^{2} \cong \frac{\left\langle N_{1}\right\rangle^{2}}{\left\langle N_{2}\right\rangle^{2}}\left[\frac{\left\langle N_{1}^{2}\right\rangle-\left\langle N_{1}\right\rangle^{2}}{\left\langle N_{1}\right\rangle^{2}}+\frac{\left\langle N_{2}^{2}\right\rangle-\left\langle N_{2}\right\rangle^{2}}{\left\langle N_{2}\right\rangle^{2}}-2 \frac{\left\langle N_{1} N_{2}\right\rangle-\left\langle N_{1}\right\rangle\left\langle N_{2}\right\rangle}{\left\langle N_{1}\right\rangle\left\langle N_{2}\right\rangle}\right] .
$$

Instead of $R$, one can use $F=Q / N$ where $Q$ is, as before, the system charge [9]. If we deal with the particles of opposite unit charges $\left(q_{1}=-q_{2}=1\right), R$ and $F$ are simply related to each other. Specifically,

$$
R=\frac{1+F}{1-F}=1+2 F+2 F^{2}+\mathcal{O}\left(F^{3}\right) .
$$

Consequently,

$$
\left\langle R^{2}\right\rangle-\langle R\rangle^{2} \cong 4\left(\left\langle F^{2}\right\rangle-\langle F\rangle^{2}\right) .
$$

Comparing Eqs. (4, 10) to each other, we get the relation

$$
\Gamma=\frac{\left\langle Z^{2}\right\rangle}{\langle N\rangle} \cong\left(q_{1}-q_{2}\right)^{2} \frac{\left\langle N_{2}\right\rangle^{4}}{\langle N\rangle^{3}}\left[\left\langle R^{2}\right\rangle-\langle R\rangle^{2}\right]=\left(q_{1}-q_{2}\right)^{2} \frac{\left\langle N_{2}\right\rangle^{4}}{\langle N\rangle^{4}} D
$$

where

$$
D \stackrel{\text { def }}{=}\langle N\rangle\left[\left\langle R^{2}\right\rangle-\langle R\rangle^{2}\right]
$$

is the charge fluctuation measure advocated in [9,13]. Since Eq. (10) holds only for sufficiently small fluctuations the same is true for the relationship (11). Therefore, $D$ is independent of the particle source distribution for small fluctuations only while $\Gamma$ possesses this property for fluctuations of any size.

Another natural measure of charge fluctuations is $9.10,23]$

$$
v(Q) \stackrel{\text { def }}{=} \frac{\left\langle Q^{2}\right\rangle-\langle Q\rangle^{2}}{\langle N\rangle}=\frac{1}{\langle N\rangle}\left[q_{1}^{2}\left(\left\langle N_{1}^{2}\right\rangle-\left\langle N_{1}\right\rangle^{2}\right)+q_{2}^{2}\left(\left\langle N_{2}^{2}\right\rangle-\left\langle N_{2}\right\rangle^{2}\right)+2 q_{1} q_{2}\left(\left\langle N_{1} N_{2}\right\rangle-\left\langle N_{1}\right\rangle\left\langle N_{2}\right\rangle\right)\right],
$$

which is not simply related to $\Gamma$, except in the two special cases: when $\langle Q\rangle=0$ and when $N$ is fixed. Then, $\Gamma=v(Q)$. One also observes that for the poissonian distribution of $N_{1}$ and $N_{2}$ there is a relation:

$$
\Gamma=v(Q)-\frac{\langle Q\rangle^{2}}{\langle N\rangle^{2}}
$$

which will be used in Sec. V to discuss the PHENIX data [23].

One more measure has been proposed in the experimental study [24]. Namely,

$$
\nu \stackrel{\text { def }}{=}\left\langle\left(\frac{N_{1}}{\left\langle N_{1}\right\rangle}-\frac{N_{2}}{\left\langle N_{2}\right\rangle}\right)^{2}\right\rangle=\frac{\left\langle N_{1}^{2}\right\rangle-\left\langle N_{1}\right\rangle^{2}}{\left\langle N_{1}\right\rangle^{2}}+\frac{\left\langle N_{2}^{2}\right\rangle-\left\langle N_{2}\right\rangle^{2}}{\left\langle N_{2}\right\rangle^{2}}-2 \frac{\left\langle N_{1} N_{2}\right\rangle-\left\langle N_{1}\right\rangle\left\langle N_{2}\right\rangle}{\left\langle N_{1}\right\rangle\left\langle N_{2}\right\rangle} .
$$

The measure $\nu$, which is computed in the poissonian approximation (5), has been called the 'statistical' contribution and denoted as $\nu_{\text {stat }}$;

$$
\nu_{\text {stat }}=\frac{1}{\left\langle N_{1}\right\rangle}+\frac{1}{\left\langle N_{2}\right\rangle}=\frac{\langle N\rangle}{\left\langle N_{1}\right\rangle\left\langle N_{2}\right\rangle} .
$$

The authors of 24] has also used the ratio $\nu / \nu_{\text {stat }}$ and the difference

$$
\nu_{\mathrm{dyn}}=\nu-\nu_{\mathrm{stat}}
$$

which they call the 'dynamical' contribution. 
Comparing Eqs. (2, (1,9) to Eqs. (14, 15, 16), one finds that for $q_{1}=-q_{2}=1$

$$
\begin{gathered}
\Gamma=4 \frac{\left\langle N_{1}\right\rangle^{2}\left\langle N_{2}\right\rangle^{2}}{\langle N\rangle^{3}} \nu, \\
\overline{z^{2}}=4 \frac{\left\langle N_{1}\right\rangle^{2}\left\langle N_{2}\right\rangle^{2}}{\langle N\rangle^{3}} \nu_{\text {stat }}, \\
\Phi=2 \frac{\left\langle N_{1}\right\rangle\left\langle N_{2}\right\rangle}{\langle N\rangle}\left(\sqrt{\frac{\nu}{\langle N\rangle}}-\sqrt{\frac{\nu_{\text {stat }}}{\langle N\rangle}}\right) \cong \frac{\left\langle N_{1}\right\rangle^{3 / 2}\left\langle N_{2}\right\rangle^{3 / 2}}{\langle N\rangle^{2}} \nu_{\text {dyn }} .
\end{gathered}
$$

The second approximate equality in Eq. (19) holds for $\nu_{\text {stat }} \gg \nu_{\text {dyn }}$.

At the end of this section we remark that

$$
\Gamma=v(Q)=\frac{1}{4} D=\frac{\langle N\rangle}{4} \nu=1,
$$

when $\langle Q\rangle=0, q_{1}=-q_{2}=1$ and the poissonian approximation (5) holds. $\Phi$ and $\nu_{\mathrm{dyn}}$ vanish by definition in this case.

\section{IV. 'BACKGROUND' MODEL}

In this section we discuss a very simple model of charge fluctuations which takes into account the charge conservation and the fact that one usually observes only a fraction of all charged particles produced in nuclear collisions. We consider the charges of two values, say +1 and -1 . The multiplicities of positive (negative) particles are denoted here as $N_{+}$ $\left(N_{-}\right)$. At the beginning we assume that all charged particles are observed. Then, $N_{+}-N_{-}=Q$, where $Q$ denotes the electric, baryonic or any other conserved charge which is the same for all events under consideration. The multiplicity distribution of negative and positive particles can be written as

$$
P_{N_{+} N_{-}}=P_{N_{-}} \delta_{N_{+}}^{N_{-}+Q},
$$

or

$$
P_{N_{+} N_{-}}=P_{N_{+}} \delta_{N_{-}}^{N_{+}-Q}
$$

Using the distribution (20) one immediately finds

$$
\begin{aligned}
\left\langle N_{+}\right\rangle & =\left\langle N_{-}\right\rangle+Q, \\
\left\langle N_{+}^{2}\right\rangle-\left\langle N_{+}\right\rangle^{2} & =\left\langle N_{-}^{2}\right\rangle-\left\langle N_{-}\right\rangle^{2}, \\
\left\langle N_{+} N_{-}\right\rangle-\left\langle N_{+}\right\rangle\left\langle N_{-}\right\rangle & =\left\langle N_{-}^{2}\right\rangle-\left\langle N_{-}\right\rangle^{2} .
\end{aligned}
$$

Then, Eqs. (2,3) give

$$
\begin{gathered}
\overline{z^{2}}=4 \frac{\left\langle N_{-}\right\rangle\left(\left\langle N_{-}\right\rangle+Q\right)}{\langle N\rangle^{2}}, \\
\Gamma=\frac{\left\langle Z^{2}\right\rangle}{\langle N\rangle}=\frac{4 Q^{2}}{\langle N\rangle^{3}}\left(\left\langle N_{-}^{2}\right\rangle-\left\langle N_{-}\right\rangle^{2}\right),
\end{gathered}
$$

where $N \equiv N_{+}+N_{-}=2 N_{-}+Q$. For $Q=0$ one gets $\overline{z^{2}}=1,\left\langle Z^{2}\right\rangle /\langle N\rangle=0$ and $\Phi=-1$.

Now, we consider a situation when only a fraction of charged particles produced in nuclear collision is observed. As many other authors, see e.g. 31, we assume that every particle is registered with the probability $p$ which is the same for all particles independently of their momenta. The particle is lost with the probability $(1-p)$. In this way, we model both the effect of tracking inefficiency and inefficiency and inefficiency and incomplete detector acceptance. Since the number of observed particles is given by the binomial distribution the multiplicity distribution of observed particles reads 


$$
\mathcal{P}_{N_{+} N_{-}}=\sum_{M_{+}=N_{+}}^{\infty} \sum_{M_{-}=N_{-}}^{\infty} P_{M_{+} M_{-}}\left(\begin{array}{c}
M_{+} \\
N_{+}
\end{array}\right) p^{N_{+}}(1-p)^{M_{+}-N_{+}}\left(\begin{array}{c}
M_{-} \\
N_{-}
\end{array}\right) p^{N_{-}}(1-p)^{M_{-}-N_{-}},
$$

where $M_{ \pm}$corresponds to the produced while $N_{ \pm}$to the observed particles.

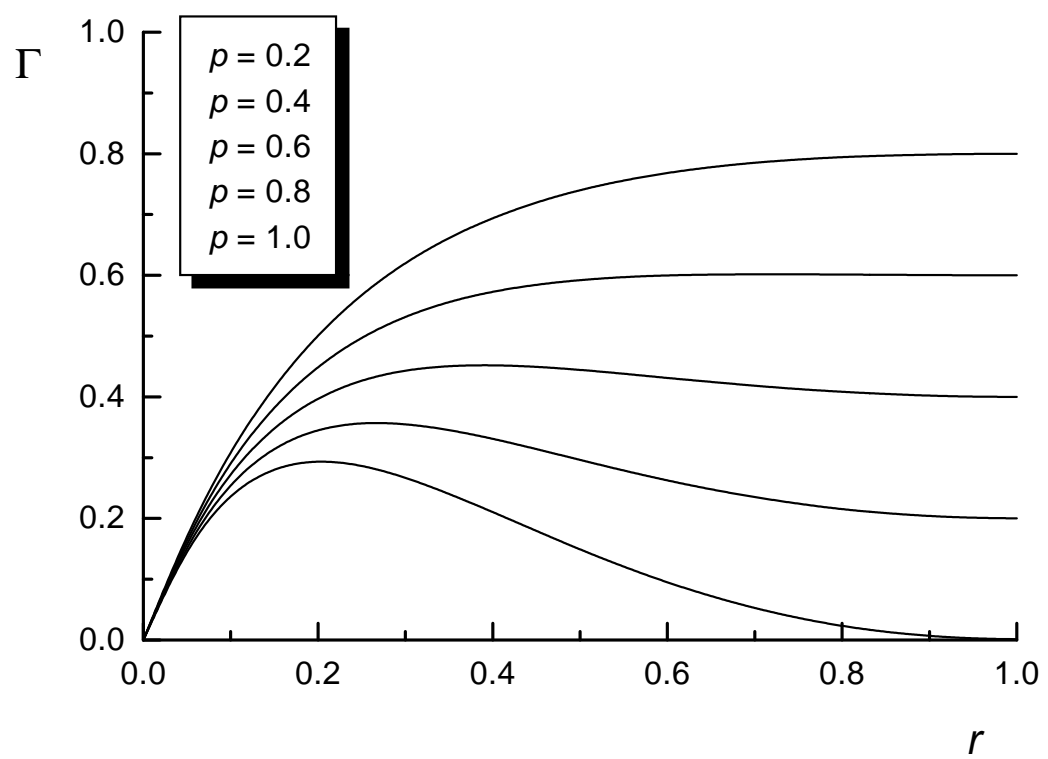

FIG. 1. $\Gamma$ as a function of the multiplicity ratio $r \equiv\left\langle N_{-}\right\rangle /\left\langle N_{+}\right\rangle$for several values of $p$. The top line corresponds to $p=0.2$, the next one to $p=0.4$, etc.

Substituting the distribution (20) into (22) one gets

$$
\begin{aligned}
\left\langle N_{-}\right\rangle & =p\left\langle M_{-}\right\rangle, \\
\left\langle N_{+}\right\rangle & =p\left\langle M_{-}\right\rangle+p Q, \\
\left\langle N_{-}^{2}\right\rangle-\left\langle N_{-}\right\rangle^{2} & =p^{2}\left(\left\langle M_{-}^{2}\right\rangle-\left\langle M_{-}\right\rangle^{2}\right)+\left(p-p^{2}\right)\left\langle M_{-}\right\rangle, \\
\left\langle N_{+}^{2}\right\rangle-\left\langle N_{+}\right\rangle^{2} & =p^{2}\left(\left\langle M_{-}^{2}\right\rangle-\left\langle M_{-}\right\rangle^{2}\right)+\left(p-p^{2}\right)\left(\left\langle M_{-}\right\rangle+Q\right), \\
\left\langle N_{+} N_{-}\right\rangle-\left\langle N_{+}\right\rangle\left\langle N_{-}\right\rangle & =p^{2}\left(\left\langle M_{-}^{2}\right\rangle-\left\langle M_{-}\right\rangle^{2}\right) .
\end{aligned}
$$

Our further considerations are limited to the poissonian approximation. Namely, one assumes that $P_{N_{-}}$in (20) is a Poisson distribution as in a classical hadron gas in equilibrium. Then, one gets

$$
\begin{aligned}
\left\langle N_{-}^{2}\right\rangle-\left\langle N_{-}\right\rangle^{2} & =\left\langle N_{-}\right\rangle, \\
\left\langle N_{+}^{2}\right\rangle-\left\langle N_{+}\right\rangle^{2} & =\left\langle N_{+}\right\rangle-p^{2} Q, \\
\left\langle N_{+} N_{-}\right\rangle-\left\langle N_{+}\right\rangle\left\langle N_{-}\right\rangle & =p\left\langle N_{-}\right\rangle .
\end{aligned}
$$

One can see here that the multiplicity distribution of negative particles is poissonian but that of positive ones is not. Substituting the above formulas to Eqs. (2,3) one gets

$$
\begin{aligned}
\overline{z^{2}} & =\frac{4 r}{(1+r)^{2}}, \\
\Gamma & =\frac{4 r}{(1+r)^{2}}-4 p \frac{r^{2}(3-r)}{(1+r)^{3}} .
\end{aligned}
$$

where $r \equiv\left\langle N_{-}\right\rangle /\left\langle N_{+}\right\rangle$. When the system is symmetric i.e. $Q=0$ and consequently $r=1$, the formulas (23, 24) simplify to

$$
\overline{z^{2}}=1, \quad \Gamma=1-p,
$$


and give

$$
\Phi=\sqrt{1-p}-1
$$

We note here that Eq. (25) holds not only for the poissonian approximation but for any distribution (20) with $Q=0$.

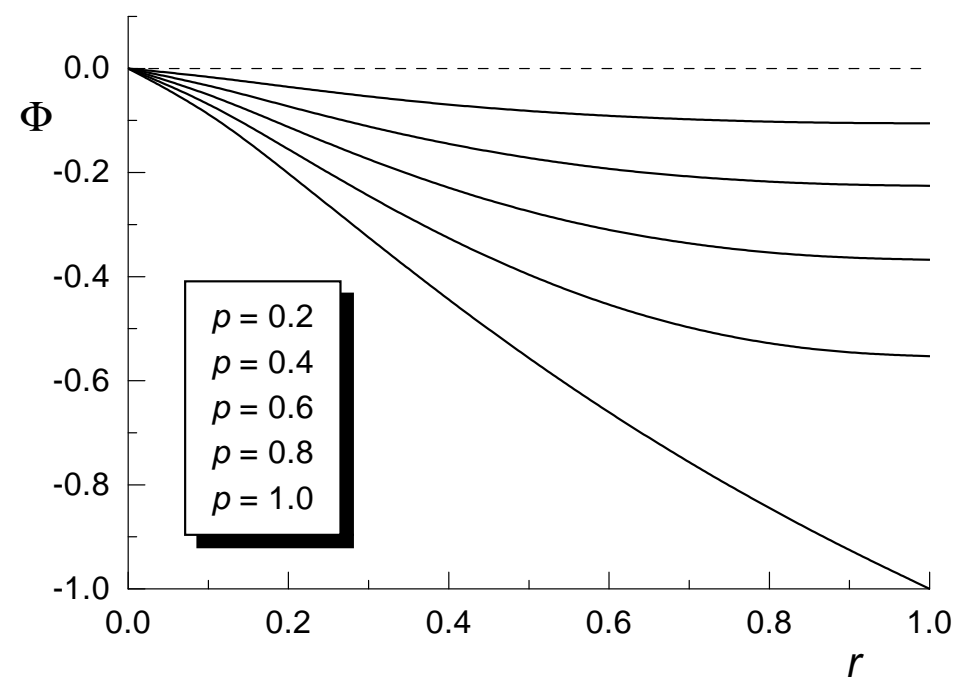

FIG. 2. $\Phi$ as a function of the multiplicity ratio $r \equiv\left\langle N_{-}\right\rangle /\left\langle N_{+}\right\rangle$for several values of $p$. The top (solid) line corresponds to $p=0.2$, the next one to $p=0.4$, etc.

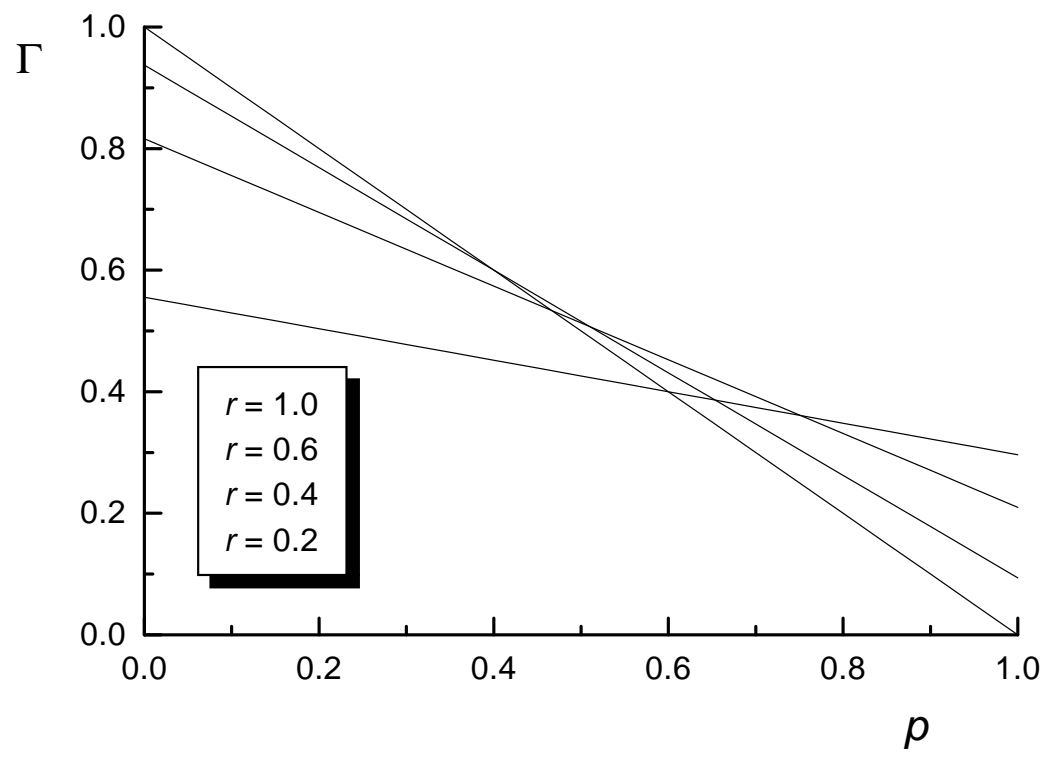

FIG. 3. $\Gamma$ as a function of the particle registration probability $p$ for several values of $r \equiv\left\langle N_{-}\right\rangle /\left\langle N_{+}\right\rangle$. The steepest line corresponds to $r=1.0$, the second steepest to $r=0.6$, etc.

The poissonian approximation can also be implemented in such a way that $P_{N_{+}}$in (21) is a Poisson distribution. Then, the multiplicity distribution of positive particles is poissonian while that of the negative ones is not. In this case the final results are 


$$
\begin{aligned}
\overline{z^{2}} & =\frac{4 r}{(1+r)^{2}}, \\
\Gamma & =\frac{4 r}{(1+r)^{2}}-4 p \frac{3 r-1}{(1+r)^{3}} .
\end{aligned}
$$

Obviously, the results (24) and (27) differ from each other. However, one observes that Eq. (24) holds for $Q \geq 0$ $(r \leq 1)$ while Eq. (27) for $Q \leq 0(r \geq 1)$. Otherwise the multiplicity distributions which are assumed to be poissonian cannot be poissonian because they must vanish for $N_{ \pm}<|Q|$. One further observes that Eqs. (23, 24) changes into Eqs. (26, 27) under the transformation $r \rightarrow 1 / r$. We also note that our final results i.e. Eqs. (23, 24) or Eqs. (26, 27) depend on $Q$ only through $r$. Therefore, the initial assumption that $Q$ is the same for all events can be relaxed and the events of different $Q$ but of the same $r$ can be combined.

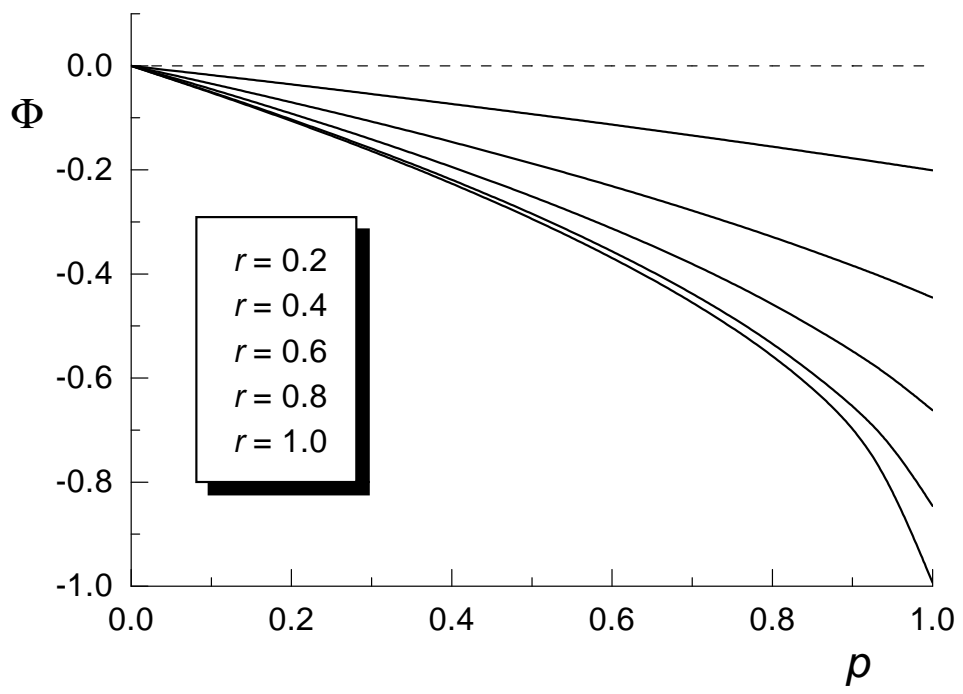

FIG. 4. $\Phi$ as a function of the particle registration probability $p$ for several values of $r \equiv\left\langle N_{-}\right\rangle /\left\langle N_{+}\right\rangle$. The top (solid) line corresponds to $r=0.2$, the next one to $r=0.4$, etc.

The $\Gamma$ - and $\Phi$-measure given by Eqs. 23, 24) are shown in Figs. 1-4. As seen, the fluctuations measured by $\Gamma$ are suppressed by the charge conservation when $p \rightarrow 1$. However, the suppression is complete for $r=1$ only. When the net charge is nonzero $(r<1)$ the fluctuations occur even at $p=1$. The same is true for the measures $D$ and $\nu$. Within the 'background' model $\Phi$ is determined by charge conservation. Therefore, the absolute value of $\Phi$ is the largest when $p=1$ and $r=1$. Then, the system is constrained most effectively. When $r \rightarrow 0$ and $p \rightarrow 0$ the effect of charge conservation is diluted.

Experimental data are often contaminated by particles coming from secondary interactions in the detector material or, in general, from sources which are different than the interactions under study. These background particles, which are not influenced by charge conservation discussed above, should be also included in our 'background' model. We assume that multiplicity distributions of both positive and negative background particles are poissonian and that their average multiplicities equal to each other. Then, one finds that Eq. (23) remains unchanged while Eq. (24) is modified as

$$
\Gamma=\frac{4 r}{(1+r)^{2}}-4 p \frac{r^{2}(3-r-2 b)}{(1+r)^{3}}
$$

where $b$ is the fraction of background particles among all negative ones. When $r=1$ Eq. (28) simplifies to $\Gamma=$ $1-p(1-b)$.

Using Eqs. (17, 18) and $(23,24)$ one finds that the ratio $\nu / \nu_{\text {stat }}$ studied by the STAR collaboration [24] equals

$$
\frac{\nu}{\nu_{\text {stat }}}=\frac{\Gamma}{\overline{z^{2}}}=1-p \frac{r(3-r)}{1+r} .
$$

In the next section Eqs 28,29$)$ are confronted with the experimental data. 


\section{EXPERIMENTAL DATA}

As noted in the Introduction, there are PHENIX 23] and STAR [24] measurements of the electric charge fluctuations in $\mathrm{Au}-\mathrm{Au}$ collisions at $\sqrt{s_{N N}}=130 \mathrm{GeV}$. The result reported by PHENIX collaboration is $v(Q)=$ $0.965 \pm 0.007$ (stat.) -0.019 (syst.). It corresponds to $10 \%$ most central collisions but the data show no significant centrality dependence. The measurements have been performed in the pseudorapidity, transverse momentum and azimuthal angle regions: $-0.35<\eta<0.35,0.2 \mathrm{GeV} / c<p_{T}$ and $0<\phi<\pi / 2$. The amount of background particles, which come from sources different than the $\mathrm{Au}-\mathrm{Au}$ interactions of interest, has been estimated as $20 \%$ $(b=0.2)$.

According to the preliminary STAR results the ratio $\nu / \nu_{\text {stat }}$ is within experimental errors independent of centrality and equals $0.80 \pm 0.03$ for $-0.5<\eta<0.5,0.1<p_{T}<2.0 \mathrm{GeV} / c$ and full azimuthal angle coverage. The background is of order of $1 \%$ in the case of STAR and it is further neglected in our considerations. To compare the experimental results to the 'background' model predictions one needs to estimate two parameters: $p$ and $r$.

The BRAHMS collaboration has found [32] that on average $3860 \pm 300$ charged particles (within $-4.7<\eta<4.7$ ) are produced in the most central $\mathrm{Au}-\mathrm{Au}$ collisions with 352 participants. Among these particles $553 \pm 36$ i.e. $14 \pm 2$ $\%$ appears in the interval $-0.5<\eta<0.5$. The corresponding number is $10 \pm 1.4 \%$ for $-0.35<\eta<0.35$. Using the exponential parameterization of the transverse momentum distribution $\left(\sim p_{T} e^{-p_{T} / T}\right)$, we have also estimated that about $10 \%$ and $26 \%$ of particles for STAR and PHENIX, respectively, are lost because of the low $p_{T}$ cut-off $p_{T}^{\min }=100 \mathrm{MeV} \cong T / 2$ and $p_{T}^{\min }=200 \mathrm{MeV} \cong T$. Finally, taking into account the tracking efficiency, which is about $90 \%$ for STAR and $80 \pm 5 \%$ for PHENIX, we have obtained $p=0.11 \pm 0.02$ (STAR) and $p=0.015 \pm 0.003$ (PHENIX). In the case of PHENIX, an additional factor 0.25 has been included due to the limited azimuthal coverage.

As already mentioned, according to the BRAHMS data [32], there is $3860 \pm 300$ charged particles produced by 352 participants. This number corresponds, on average, to 140 protons and 212 neutrons in $\mathrm{Au}$-Au collisions. Therefore, $\left\langle N_{+}\right\rangle=2000 \pm 150$ and $\left\langle N_{-}\right\rangle=1860 \pm 150$. Consequently, $r=\left\langle N_{-}\right\rangle /\left\langle N_{+}\right\rangle=0.93 \pm 0.14$.

Substituting the estimated values of $p$ and $r$ into Eqs. (28,29) and using Eq. (13) one finds $v(Q)=0.990 \pm 0.003$ for PHENIX and and $\nu / \nu_{\text {stat }}=0.89 \pm 0.02$ for STAR. Both theoreical estimates are somewhat higher than the experimental values: $0.965+0.007-0.026$ (PHENIX) and $0.80 \pm 0.03$ (STAR). The small differences are presumably due to the neutral resonances which decay into charge hadrons and effectively reduce the charge fluctuations [9, 10]. As shown by Zaranek [29], the effect of resonances strongly depends on the rapidity window, where the charge hadrons are observed, and the fluctuations can be even enhanced in sufficiently small windows. In any case, we conclude this section by saying that the model of a classical hadron gas in equilibrium approximately explains the experimentaly observed electric charge fluctuations and there is not much space for dynamical effects.

\section{BACKGROUND FREE MEASURES}

Since the effects of charge conservation and incomplete acceptance are of no real interest, it is desirable to use such measures which are insensitive to both effects. The authors of Ref. 13] introduced the modified $D$-measure which in our notation equals

$$
\tilde{D}=\frac{1}{r^{2}(1-p)} D
$$

where $D$ is defined by Eq. (12). Using the relation (11) and Eq. (24) one finds $\tilde{D}$ corresponding to the 'background' model. It equals

$$
\tilde{D}=\frac{(1+r)^{2}}{r(1-p)}\left[1-p \frac{r(3-r)}{1+r}\right]
$$

As seen in Eq. (30), $\tilde{D} \neq 4$ for $r \neq 1$. Figs. 5 and 6 , where Eq. (30) is illustrated, show that the difference can be significant. A similar conclusion has been recently drawn by Zaranek 29] who has studied how $\tilde{D}$ behaves in variety of simple fluctuation models. He has also proposed another background-free measure $\Delta \Phi=\Phi-\Phi_{0}$ with $\Phi_{0}$ given by Eq. (25) which holds for $Q=0$. Since one often deals with the systems where $Q>0$ it would be preferable to use as $\Phi_{0}$ the expression given by Eqs. (23,24).

When the electric charge fluctuations are studied, $r$ is close to unity at sufficiently high collision energies because the multiplicity of the produced charged hadrons is significantly larger than the number of participating protons. Then, the $(1-p)$ correction [13] works well. At lower energies, $r$ significantly differs from 1 . The ratio is also noticeably 
smaller than unity when the baryon number fluctuations are studied. Then, one can use our 'background' model to construct a variety of the background free measures. For example, $\Gamma-\Gamma_{0}$ or $\Gamma / \Gamma_{0}$, where $\Gamma_{0}$ is given by Eq. (24).

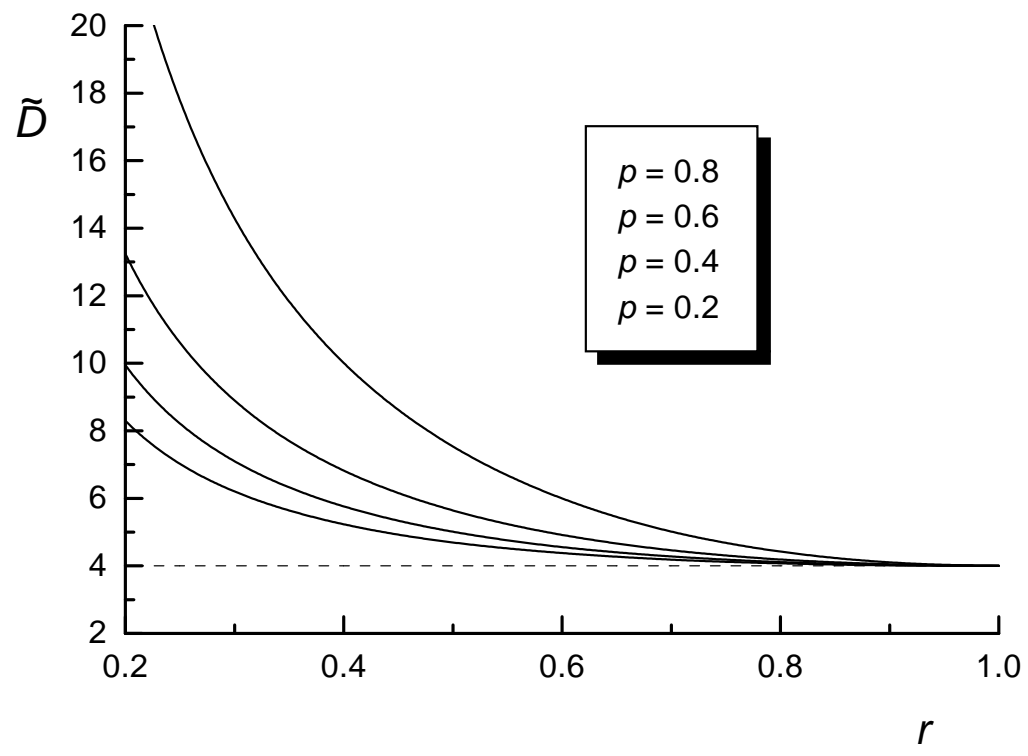

FIG. 5. $\tilde{D}$ as a function of the multiplicity ratio $r \equiv\left\langle N_{-}\right\rangle /\left\langle N_{+}\right\rangle$for several values of $p$. The top line corresponds to $p=0.8$, the next one to $p=0.6$, etc.

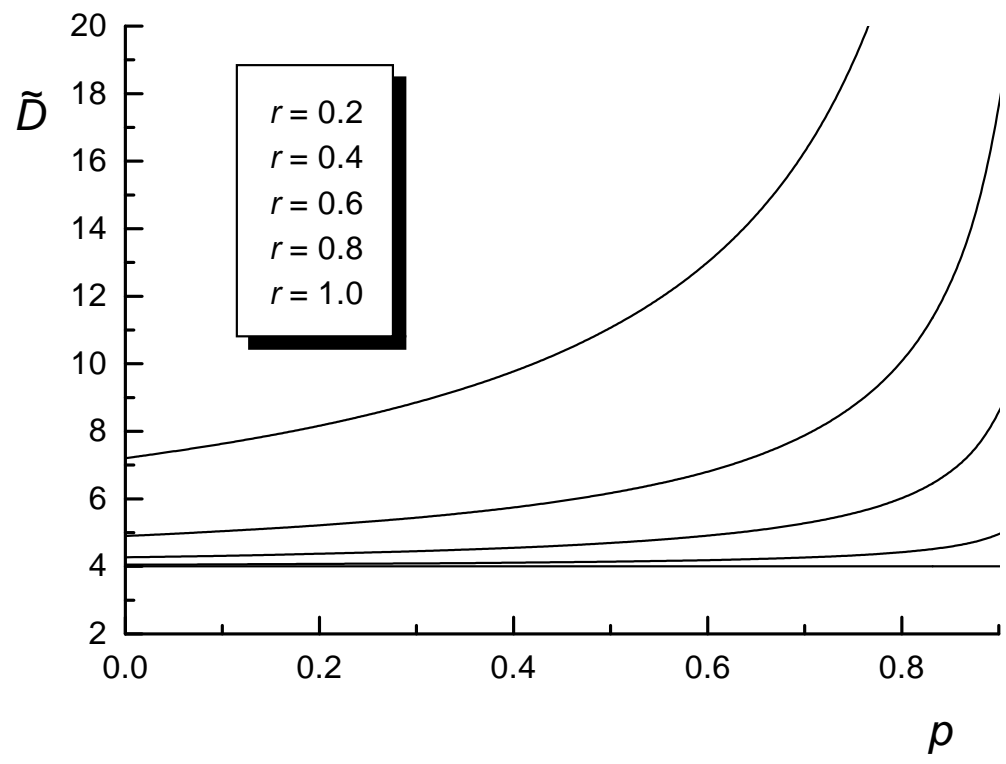

FIG. 6. $\tilde{D}$ as a function of the particle registration probability $p$ for several values of $r \equiv\left\langle N_{-}\right\rangle /\left\langle N_{+}\right\rangle$. The top line corresponds to $r=0.2$, the next one to $r=0.4$, etc. 


\section{SUMMARY AND CONCLUSIONS}

We have shown that the $\Phi$-measure, which has been successfully applied to the transverse momentum fluctuations, can be also used to study charge fluctuations. $\Phi$ is insensitive to the collision centrality and sensitive to the dynamics. If one is interested not only in the dynamical fluctuations but in the absolute value the fluctuations the $\Gamma-$ measure, which is related to $\Phi$, can be applied. We have established the relationships between $\Phi, \Gamma$ and several charge fluctuation measures proposed by other authors. The measure $D$ [9, 13] has been shown to be equivalent to $\Gamma$ but for small fluctuations only.

The charge fluctuations have been analyzed within a 'background' model where the particles are produced statistically being constrained by charge conservation. The effects of the finite acceptance of experimental apparatus and of the detection inefficiency are schematically taken into account. The model's results depend solely on the two parameters: $p$ which is a fraction of all produced particles taken to the analysis and $r$ being the ratio $\left\langle N_{-}\right\rangle /\left\langle N_{+}\right\rangle$. The 'background' model's predictions are close to the PHENIX [23] and preliminary STAR [24] results on electrical charge fluctuations. Thus, there is little space for the dynamical effects such as freezing of the fluctuations generated at the quark-gluon plasma phase [9,10].

At the end we have considered the measures which are supposed to be free of the effects of charge conservation and incomplete acceptance. We have shown within our 'background' model that when $r$ is noticeably smaller than unity, as in the case of the baryonic charge fluctuations, the simple corrections proposed by other authors do not work properly.

The data on the charge fluctuations [19,23,24 show that the dynamical phenomena do not contribute significantly to the observed fluctuations. Thus, a proper choice of a statistical tool for data analysis is important to quantify and interpret the small effects of interest. It is also important to carefully eliminate trivial effects as those caused by the charge conservation and non-vanishing net charge. The results presented here contribute to these goals.

\section{ACKNOWLEDGMENTS}

I am very grateful to Sergei Voloshin for the correspondence that initiated this work. I am indebted to him, Marek Gaździcki and Jacek Zaranek for critical comments on the manuscript. My thanks also go to Joakim Nystrand and Evert Stenlund for a discussion on the PHENIX data.

[1] S. Jeon and V. Koch, Phys. Rev. Lett. 83, 5435 (1999).

[2] S. Gavin and C. Pruneau, nucl-th/9907040.

[3] S. Gavin and C. Pruneau, nucl-th/9908070.

[4] D. Bower and S. Gavin, Phys. Rev. C 64, 051902 (2001).

[5] M.A. Halasz et al., Phys. Rev. D 58, 096007 (1998).

[6] M. Stephanov, K. Rajagopal and E. Shuryak, Phys. Rev. Lett. 81, 4816 (1998).

[7] J. Berges and K. Rajagopal, Nucl. Phys. B 538, 215 (1999).

[8] Z. Fodor and S.D. Katz, hep-lat/0106002.

[9] S. Jeon and V. Koch, Phys. Rev. Lett. 85, 2076 (2000).

[10] M. Asakawa, U. Heinz and B. Müller, Phys. Rev. Lett. 85, 2072 (2000).

[11] K. Fiałkowski and R. Wit, hep-ph/0006023.

[12] K. Fiałkowski and R. Wit, Europhys. Lett. 53, 184 (2001).

[13] M. Bleicher, S. Jeon and V. Koch, Phys. Rev. C 62, 061902 (2000).

[14] H. Heiselberg and A.D. Jackson, Phys. Rev. C 63, 116003 (2001).

[15] M. Gaździcki and St. Mrówczyński, nucl-th/0012094.

[16] W. Bopp and J. Ranft, Eur. Phys. J. C 22, 171 (2001).

[17] E. Shuryak and M. Stephanov, Phys. Rev. C 63, 064903 (2001).

[18] Z. Lin and C.M. Ko, Phys. Rev. C 64, 041901 (2001).

[19] G. Roland and NA49 Collaboration, Nucl. Phys. A 638, 91c (1998).

[20] S.V. Afanasiev et al., Phys. Rev. Lett. 86, 1965 (2001).

[21] G. Baym and H. Heiselberg, Phys. Lett. B 469, 7 (1999).

[22] C.B. Yang and X.Cai, Int. J. Mod. Phys. Lett. 16 (2001) 1227. 
[23] K. Adcox et al., nucl-ex/0203014.

[24] S.A. Voloshin and STAR Collaboration, nucl-ex/0109006.

[25] M. Gaździcki and St. Mrówczyński, Z. Phys. C 54, 127 (1992).

[26] H. Appelshäuser et al., Phys. Lett. B 459, 679 (1999).

[27] M. Gaździcki, Euro. Phys. J. C 8, 131 (1999).

[28] St. Mrówczyński, Phys. Lett. B 459, 13 (1999).

[29] J. Zaranek, hep-ph/0111228.

[30] St. Mrówczyński, Phys. Lett. B 465, 8 (1999).

[31] D.P. Mahapatra, B. Mohanty and S.C. Phatak, Int. J. Mod. Phys. A 17, 657 (2002).

[32] I.G. Bearden et al., Phys. Lett. B 523, 277 (2001). 